\title{
Influence of collateral circulation on cerebral blood flow and frontal lobe cognitive function in patients with severe internal carotid artery stenosis
}

\author{
Wei Wei ${ }^{1}$, Xingyang $\mathrm{Yi}^{2}$, Jianghai Ruan ${ }^{1}$, Xiaodong Duan ${ }^{3}$, Hua Luo ${ }^{1 *}$ and Zhiyu LV ${ }^{1}$
}

\begin{abstract}
Background: This study aimed to investigate the cerebral blood flow (CBF) and frontal lobe cognitive function in severe internal carotid artery (ICA) stenosis patients with different types of collateral circulation.

Methods: One hundred twenty-six patients with severe unilateral ICA stenosis were enrolled. Digital subtraction angiography (DSA) was performed to recruit patients with one of three common types of collateral circulation: anterior communicating artery $(A \subset \mathrm{A})$, posterior communicating artery (PCOA) and ophthalmic artery $(\mathrm{OA})$. The hemodynamic parameters of the middle cerebral artery (MCA) were measured using transcranial Doppler (TCD), and the individual frontal lobe cognitive attention functions were evaluated using Word Fluency Test, Trail-Making Test (TMT), Digit Span, and Stroop Color Word Test (SCWT). The correlation between hemodynamic changes and the scores of all tasks was analyzed.

Results: On the side of arterial stenosis, the CBF velocities were highest in AcoA group and lowest in the OA group. All patients performed worse in TMT and Digit Span than the matched normal controls. The AcoA group exhibited a lower pulsatility index (PI) and a longer response time in the Stroop task, but had a higher accuracy rate in the Stroop task and higher scores in Word Fluency Test than the PcoA and OA groups. In all the three groups, PI was positively correlated with the accuracy rate for Stroop interference effects.

Conclusions: Our findings suggested that the frontal lobe cognitive function of patients with ICA was impaired, and AcoA collaterals may be beneficial for selective attention functions, whereas OA collaterals may be associated with impairment of selective attention functions. Additionally, a high PI may be an indicator for identifying impaired selective attention in patients with severe ICA stenosis.
\end{abstract}

Keywords: Internal carotid artery, Stenosis, Collateral circulation, Cerebral blood flow, Cognitive function

\section{Background}

Severe internal carotid artery (ICA) stenosis is characterized by chronic cerebral hypoperfusion due to the decreased cerebral blood flow (CBF). It has been proven that longlasting cerebral hypoperfusion may impair energy metabolism in neurons and lead to cognitive impairment $[1,2]$. Although cerebral hypoperfusion is common in patients with ICA stenosis, collateral circulation provides new paths

\footnotetext{
*Correspondence: Izlh@sina.com

'Department of Neurology, the Affiliated Hospital of Southwest Medical

University, No. 25 Taiping Road, Luzhou 646000, Sichuan, China

Full list of author information is available at the end of the article
}

to maintain sufficient blood supply even though it may only represent a poor compensation [2]. Some studies have investigated the association between collateral circulation and cognitive dysfunction in recent years; however, the findings on this topic have been inconsistent [3, 4]. Everts et al. found no correlation between the formation of collaterals and cognitive function [5], while Sztriha et al. proposed that collaterals-related perfusion restoration could improve the cognitive dysfunction [6]. Thus far, there has not yet been a study investigating the specific association between different types of collateral circulation and cerebral

(c) The Author(s). 2019 Open Access This article is distributed under the terms of the Creative Commons Attribution 4.0 International License (http://creativecommons.org/licenses/by/4.0/), which permits unrestricted use, distribution, and 
hemodynamics as well as cognitive function in patients with severe ICA stenosis.

Word Fluency Test is a sensitive measure for frontal lobe cognitive functions [7], and Trail-Making Test (TMT) is frequently used in neuropsychological assessments to estimate executive function [8]. Forward and Reverse Digit Span tests evaluate working memory. Stroop Color Word Test (SCWT) is a classic, widely-used psychological evaluation tool that ignores irrelevant information and enhances the ability of suppressing interference which delays the response [9]. It demands resolution of a conflict reading and naming, which require selective attention [10]. Furthermore, selective attention refers to a top-down information processing to selectively filter information, which can be adjusted by enhancement or inhibition of neural activities associated with pulvino-cortical networks [11] and dorsolateral prefrontal cortex [12]. Collectively, the aforementioned tests call for the function of frontal lobe, which could be influenced by the blood supply of ICA.

Thus, we hypothesized that patients with severe ICA stenosis may show different behavioral performance in frontal lobe cognitive functions due to diverse collateral compensations. The present study aimed to investigate the cerebral blood flow (CBF) and frontal lobe cognitive function in severe ICA stenosis patients with different types of collateral circulation.

\section{Methods}

\section{Participants}

This study was approved by the Ethics Committee of the Affiliated Hospital of Southwest Medical University. Written informed consent was obtained from each participant.

A total of 126 patients with severe unilateral ICA stenosis were enrolled between January 2013 and October 2017. The diagnosis of ICA stenosis was confirmed by digital subtraction angiography (DSA) (stenosis $\geq 70 \%$ ) according to the North American Symptomatic Carotid Endarterectomy Trail (NASCET) criteria [13]. Patients with a single type of collateral circulation: anterior communicating artery (AcoA), posterior communicating artery (PcoA), or ophthalmic artery (OA) confirmed by DSA were selected. The exclusion criteria included: 1 ) dementia (Montreal Cognitive Assessment < 26) [14]; 2) disturbance of consciousness; 3) transient ischemic attack (TIA); 4) MRI showing cerebral infarction or other pathological brain diseases, such as hemorrhages, tumors, aneurysms and vascular anomalies; 5) inadequate temporal window in TCD examination; 6) concomitant stenosis in other arteries; or 7) color blindness or color weakness. According to the type of collateral circulation, patients were classified into three groups: AcoA group, PcoA group, and OA group. Eighty-one control participants matched for age, sex, and education, were enrolled from the individuals who underwent DSA with normal results. The exclusion criteria were identical to those applied for ICA stenosis patients.

\section{TCD examination}

Blood flow velocity (BFV) of the ipsilateral MCA was measured at a depth of $50 \mathrm{~mm}$ by TCD, placing a handheld transducer over the temporal bone, using a $2-\mathrm{MHz}$ TCD device (EMS-9A, Delica, China). The BFV parameters included systolic flow velocity (Vs), diastolic flow velocity $(\mathrm{Vd})$ and mean flow velocity $(\mathrm{Vm})$. The pulsatility index $(\mathrm{PI})$ was calculated as follows: $\mathrm{PI}=(\mathrm{Vs}-\mathrm{Vd}) / \mathrm{Vm}$.

\section{Neuropsychological evaluation}

The Word Fluency Test required participants to give vegetable words within 1 min [15]. TMT was composed by Part A and Part B [8]. Part A requires participants to draw a line connecting 25 encircled number distributed in a piece of paper consecutively; Part B requires participants to connect the alternating encircled numbers and letters consecutively. It was rated by the time used in finishing each part. Digit Span required participants to repeat a series of increased length of numbers in forward and reverse order [16].

Selective attention was evaluated using SCWT. Four color names in Chinese (red, yellow, green, and blue) were presented on the computer screen. First, the name of one color was presented in black, and the subject was instructed to respond by pressing the corresponding key (SCWT A). Second, patches colored in one of these colors were presented, which was judged by the subject (SCWT B). Third, the color of the word did not match the meaning of the word, and the subject was requested to judge the color ignoring the incongruent meaning (SCWT C). Each subtask contains 50 stimuli and should be completed as fast as possible. The response time (RT) and accuracy rate (AR) were recorded. RT for Stroop interference effects (SIE) was calculated as "RT $\mathrm{SCWT}_{\mathrm{C}}-\mathrm{RT}_{\text {SCWT }} \mathrm{B}$ " . The AR for SIE was calculated as "AR $\mathrm{R}_{\text {SCWT }}$ B- $\mathrm{AR}_{\text {SCWT C" }}$ " [17]. Each subject was provided the explanation of SCWT, and practiced the paradigm a trial of 18 rounds (six rounds per subtask).

\section{Statistical analysis}

All statistical analyses were performed using SPSS software (Version 24.0, SPSS Inc., Chicago, IL, USA). Continuous variables were expressed in median and interquartile values. Chi-squared test was used for the analyses of categorical variables, and non-parametric Kruskal-Wallis and post hoc analysis were applied for continuous variables. Correlation between the BFV parameters and neuropsychological scores were analyzed using Spearman's correlation analysis. Probability $(P)$ values $\leq 0.05$ were considered statistically significant. 


\section{Result}

\section{Clinical characteristics}

Among the 126 patients with severe unilateral ICA stenosis, 105 patients and all the normal controls completed all the examinations and tests. Based on their results, 65 patients were assigned to the AcoA group, 28 patients to the PcoA group, and 12 patients to the OA group. Demographic data and risk factors were summarized (Table 1). There was no significant difference in these demographic characteristics and risk factors among the four groups (all $P>0.05$ ). In the AcoA group, the incidence of diabetes mellitus $\left(\chi^{2}=4.125, P=0.042\right)$ and hyperlipidemia $\left(\chi^{2}=5.395, P=0.02\right)$ were slightly higher than those in controls after pairwise comparisons.

\section{Influence of collateral circulation on BFV}

The ipsilateral BFV parameters (Vs, $\mathrm{Vm}$, and $\mathrm{Vd}$ ) in patients with ICA stenosis were significantly lower than those in normal controls $(P<0.0001)$. These BFV parameters from high to low in order were AcoA group $>$ PcoA group > OA group (all $P<0.05$ ). PI in patients was significantly lower than that in normal controls, and PI from high to low in order was OA group $>$ PcoA group $>$ AcoA group (all $P<0.05$ ). The statistical results were presented in Table 2. The contralateral BFV parameters (Vs, Vm, and $\mathrm{Vd}$ ) only in the AcoA group were significantly higher than those in the other groups (all $P<0.0001$ ). There was no significant difference in contralateral PI among the groups $(P>0.05)$ (Table 3$)$.

\section{Influence of collateral circulation on neuropsychological tests}

Word Fluency Test scores of the AcoA group and controls were significantly higher than those in the PcoA and OA groups (AcoA vs PcoA, $P=0.005$; AcoA vs OA, $P=0.012$; PcoA vs controls, $P<0.0001$; OA vs controls, $P=0.001$ ), and there was no significant difference between the AcoA group and controls. All patients required significantly longer time to finish TMT (Part A
Table 2 The $P$ values of ipsilateral BFV parameters between different groups

\begin{tabular}{lllll}
\hline & Vs & $V m$ & $V d$ & $P l$ \\
\hline AcoA vs PcoA & 0.017 & $<0.0001$ & $<0.0001$ & 0.034 \\
AcoA vs OA & $<0.0001$ & $<0.0001$ & $<0.0001$ & 0.032 \\
AcoA vs controls & $<0.0001$ & $<0.0001$ & 0.036 & $<0.0001$ \\
PCOA vs OA & 0.003 & 0.002 & 0.007 & 0.997 \\
PcoA vs controls & $<0.0001$ & $<0.0001$ & $<0.0001$ & $<0.0001$ \\
OA vs controls & $<0.0001$ & $<0.0001$ & $<0.0001$ & $<0.0001$
\end{tabular}

Vs, stolic flow velocity; Vm, mean flow velocity; Vd, diastolic velocity; PI, pulsatility index. $P$ value, after post Hoc tests

and Part B) than controls (for Part A: AcoA vs controls, $P=$ 0.013; PcoA vs controls: $P=0.018$; OA vs controls, 0.023 ; for Part B: AcoA vs controls, $P<0.0001$; PcoA vs controls, $P=$ 0.002 ; OA vs controls, $P=0.001$ ). The scores of Digit Span (forward and reverse) in all patients were significantly lower than those of the control participants (for forward: AcoA vs controls, $P=0.01$; PcoA vs controls, $P=0.009$; OA vs controls, $P=0.016$; for backward: every patient group vs controls, $P<0.0001)$. There was no significant difference among the three patient groups in TMT (Part $\mathrm{A}$ and Part B) and Digit Span $(P>0.05)$ (Table 4).

There was no significant difference in $\mathrm{RT}_{\mathrm{SCWT}} \mathrm{A}$ or $\mathrm{RT}_{\text {SCWT }}$ в among the four groups $(P>0.05)$. $\mathrm{RT}_{\text {SCWT C }}$ and RT for SIE in all patient groups were significantly longer than those in normal controls (for SCWTC: AcoA vs controls, $P<0.0001$; PcoA vs controls, $P=$ 0.002; OA vs controls, $P=0.043$; for RT for SIE: AcoA vs controls, $P<0.0001$; PcoA vs controls, $P=0.021$; OA vs controls, $P=0.005$ ), and the AcoA group had higher score than the PcoA groups (for SCWT C: $P=0.001$; for RT for SIE: $P=0.02$ ) and OA groups (for SCWT C: $P=0.046$; for RT for SIE: $P=0.03$ ). There was no significant difference in $\mathrm{AR}_{\mathrm{SCWT}}$ A or $\mathrm{AR}_{\mathrm{SCWT}}$ в among the four groups $(P>0.05)$. $\mathrm{AR}_{\mathrm{SCWT}} \mathrm{C}$ in the OA group was significantly lower than that in the normal controls $(P=0.004)$, while there was no significant difference in

Table 1 Demographic data of patients with severe ICA stenosis and normal controls

\begin{tabular}{|c|c|c|c|c|c|c|}
\hline Characteristics & AcoA group & PcoA group & OA group & Controls & $\mathrm{H}$ or $x^{2}$ & $P$ value \\
\hline Number & 65 & 28 & 12 & 81 & & \\
\hline Age (years) & $68.00(60.50,72.00)$ & $67.50(62.25,71.75)$ & $62.00(59.00,68.25)$ & $69.00(62.00,72.00)$ & 6.684 & 0.083 \\
\hline $\operatorname{Sex}(M / F)$ & $47 / 18$ & $18 / 10$ & $8 / 4$ & $53 / 28$ & 1.420 & 0.701 \\
\hline Education (years) & $8.00(5.00,12.00)$ & $8.50(5.00,12.00)$ & $7.50(3.75,10.75)$ & $6.00(3.50,10.00)$ & 3.158 & 0.368 \\
\hline Hypertension & $48(73.8 \%)$ & 19 (67.9\%) & $8(66.7 \%)$ & $48(59.3 \%)$ & 3.474 & 0.324 \\
\hline Diabetes mellitus & $35(53.8 \%)$ & $12(42.9 \%)$ & $6(50 \%)$ & $30(37.0 \%)$ & 4.300 & 0.231 \\
\hline Hyperlipidemia & $51(78.5 \%)$ & $20(71.4 \%)$ & $9(75 \%)$ & 49 (60.5\%) & 5.765 & 0.124 \\
\hline Smoking history & $42(64.6 \%)$ & 19 (67.9\%) & $7(58.3 \%)$ & $50(61.7 \%)$ & 0.512 & 0.916 \\
\hline Alcoholism & 10 (15.4\%) & 5 (17.9\%) & $2(16.7 \%)$ & $20(24.7 \%)$ & 2.151 & 0.542 \\
\hline
\end{tabular}

$X^{2}$ value, by Chi-squared test. $H$ and $P$ value, by non-parametric Kruscal-Wallis tests within the four groups 
Table 3 TCD parameters in patients with severe ICA stenosis and normal controls

\begin{tabular}{lllllll}
\hline Parameters & AcoA group & PcoA group & OA group & Controls & $H$ & $P$ value \\
\hline Vs (Affected side) & $50.00(44.50,56.00)$ & $44.00(36.25,52.25)$ & $31.50(27.25,41.25)$ & $79.00(74.00,85.00)$ & 143.610 & $<0.0001^{*}$ \\
Vs (Unaffected side) & $123.00(102.00,136.50)$ & $80.50(68.25,96.00)$ & $96.00(69.00,107.25)$ & & 102.901 & $<0.0001^{*}$ \\
Vm (Affected side) & $39.00(35.00,43.50)$ & $32.50(28.25,38.00)$ & $22.50(20.25,30.50)$ & $52.00(46.00,57.00)$ & 117.469 & $<0.0001^{*}$ \\
Vm (Unaffected side) & $73.00(63.00,86.50)$ & $47.50(42.25,61.50)$ & $55.50(41.25,67.25)$ & & 78.395 & $<0.0001^{*}$ \\
Vd (Affected side) & $33.00(28.00,37.00)$ & $28.00(23.25,31.75)$ & $18.50(17.00,25.50)$ & $37.00(32.75,40.00)$ & 55.753 & $<0.0001^{*}$ \\
Vd (Unaffected side) & $51.00(42.00,64.50)$ & $31.50(28.00,43.75)$ & $35.50(28.50,44.50)$ & & 57.931 & $<0.0001^{*}$ \\
Pl (Affected side) & $0.45(0.35,0.54)$ & $0.55(0.42,0.60)$ & $0.53(0.50,0.59)$ & $0.85(0.75,1.03)$ & 131.688 & $<0.0001^{*}$ \\
Pl (Unaffected side) & $0.88(0.74,1.11)$ & $0.96(0.85,1.13)$ & $0.99(0.87,1.15)$ & & 6.445 & 0.092 \\
\hline
\end{tabular}

$V s$, stolic flow velocity; $V m$, mean flow velocity; $V d$, diastolic velocity; $P I$, pulsatility index; $H$ and $P$ value, by non-parametric Kruscal-Wallis tests within the four groups

${ }^{*} P<0.0001$ indicate statistically significant differences

$\mathrm{AR}_{\text {SCWT } C}$ among AcoA group, PcoA group and normal controls $(P>0.05)$. AR for SIE in PcoA group and OA group was significantly higher than that in AcoA group (for PcoA group, $P=0.03$; for OA group, $P=0.026$ ) and controls (for PcoA group, $P=0.001$; for OA group, $P=0.015)$, which also caused differences among the four groups $(P<0.0001)$ (Table 5).

\section{Correlation between BFV parameters and neuropsychological scores}

There was no significant correlation between BFV parameters and Word Fluency Test or Digit Span. The ipsilateral PI was positively correlated with AR for SIE in patients with ICA stenosis $(P<0.05)$, especially in PcoA group and OA group $(P<0.01)$. The ipsilateral PI was positively correlated with RT for SIE in the PcoA group $(P<0.05)$. There was a negative correlation between $\mathrm{Vd}$ and $\mathrm{RT}$ for SIE in the OA group $(P<0.05)$ (Table 6) (Fig. 1).

\section{Discussion}

In the present study, we investigated whether cerebral BFV and frontal lobe cognitive function would be influenced by the different type of collateral circulation in patients with ICA stenosis. Previous studies have proposed that BFV measured by TCD was a major indicator reflecting cerebral hemodynamics [18], and BFV of MCA is the most reliable parameter [19]; herein, we measured BFV in bilateral MCA.
On the other hand, Word Fluency Test, TMT, Digit Span, and SCWT were also included in the frontal lobe cognitive function assessment [20]. Our findings indicated that SCWT was affected by collaterals more than other neurological function tests. Moreover, we found the ipsilateral PI was significantly associated with impaired selective attention. In brief, the current study provides a new insight into the correlations among collateral circulation, cerebral perfusion and selective attention.

\section{Relationship between CBFV and collateral circulation}

Generally, ICA stenosis mainly affects the blood perfusion of ipsilateral anterior circulation. We found the BFV of ipsilateral MCA was significantly reduced in patients with ICA stenosis. Especially, the decrease of BFV in PcoA group was more apparent than that in AcoA group, suggesting collateral circulation through the AcoA may provide a better compensation for the impaired cerebral perfusion. However, Gfusi et al. proposed that collateral circulation through PcoA to be important for cerebral perfusion because the absence of PcoA collaterals was found to be associated with poor prognosis [21]. Thus, we also speculate collateral circulation through the PcoA may be necessary in the absence of AcoA collaterals. Additionally, the BFV of contralateral MCA in AcoA group was higher than that in other groups, which may be attributed to the compensation.

Table 4 Comparison of cognitive assessment in patients with severe ICA stenosis and normal controls

\begin{tabular}{lllllll}
\hline & AcoA group & PcoA group & OA group & Controls & $H$ & $P$ value \\
\hline Word Fluency Test & $15.00(13.00,18.50)$ & $13.00(10.00,15.75)$ & $10.50(9.00,16.00)$ & $16.00(14.00,18.50)$ & 25.703 & $<0.0001^{*}$ \\
TMT(s) & & & & & \\
$\quad$ TMT-A & $33.51(28.61,40.23)$ & $37.89(28.68,41.58)$ & $38.50(32.22,43.54)$ & $28.82(25.00,34.63)$ & 17.850 & $<0.0001^{*}$ \\
$\quad$ TMT-B & $68.11(62.06,74.53)$ & $68.26(60.48,75.52)$ & $73.50(68.54,78.51)$ & $60.21(55.36,68.78)$ & 28.739 & $<0.0001^{*}$ \\
Digit Span & & & & \\
$\quad$ Digit Span forward & $6.00(5.00,7.00)$ & $6.00(4.25,7.00)$ & $5.00(4.25,6.75)$ & $7.00(6.00,8.50)$ & 17.742 & $<0.0001^{*}$ \\
$\quad$ Digit Span backward & $3.00(2.00,4.00)$ & $3.00(3.00,4.00)$ & $3.50(3.00,4.00)$ & $5.00(4.00,6.00)$ & 74.082 & $<0.0001^{*}$ \\
\hline
\end{tabular}

TMT, Trail-Making Test; $H$ and $P$ value, by non-parametric Kruscal-Wallis tests within the four groups

${ }^{*} P<0.001$ indicate statistically significant differences 
Table 5 SCWT assessment in patients with severe ICA stenosis and normal controls

\begin{tabular}{|c|c|c|c|c|c|c|}
\hline SCWT parameters & AcoA group & PcoA group & OA group & Controls & $\mathrm{H}$ & $P$ value \\
\hline \multicolumn{7}{|l|}{ Response time (ms) } \\
\hline SCWT A & $30.00(21.50,36.50)$ & $29.00(20.00,37.50)$ & $28.00(21.75,34.00)$ & $28.00(18.50,33.00)$ & 2.209 & 0.566 \\
\hline SCWT B & $49.00(41.00,61.50)$ & $49.00(42.25,52.75)$ & $47.50(42.00,54.25)$ & $46.00(38.50,52.50)$ & 4.842 & 0.184 \\
\hline SCWT C & $104.00(94.50,117.50)$ & $96.00(82.50,106.50)$ & $92.50(86.75,102.75)$ & $82.00(70.00,91.00)$ & 65.925 & $<0.0001^{* *}$ \\
\hline Response time for SIE & $55.00(48.00,63.00)$ & $52.00(32.00,57.00)$ & $43.50(37.75,52.25)$ & $36.00(27.50,41.00)$ & 70.771 & $<0.0001^{* *}$ \\
\hline \multicolumn{7}{|l|}{ Accuracy rate (\%) } \\
\hline SCWT A & $98.00(92.50,100.00)$ & $99.00(96.25,99.75)$ & $97.00(95.25,99.75)$ & $98.00(96.00,99.50)$ & 1.134 & 0.726 \\
\hline SCWT B & $85.00(79.00,96.50)$ & $90.00(85.00,97.75)$ & $83.50(78.75,98.25)$ & $89.00(86.00,95.00)$ & 4.924 & 0.177 \\
\hline SCWT C & $81.00(75.50,90.00)$ & $81.00(75.25,90.75)$ & $78.50(69.75,86.00)$ & $85.00(80.00,90.00)$ & 11.579 & $0.009^{*}$ \\
\hline Accuracy rate for SIE & $4.00(2.50,6.00)$ & $7.00(5.25,11.75)$ & $9.00(5.50,11.75)$ & $4.00(2.00,5.00)$ & 35.621 & $<0.0001^{* *}$ \\
\hline
\end{tabular}

$S I E$, Stroop interference effects; $H$ and $P$ value, by non-parametric Kruscal-Wallis tests within the four groups

${ }^{*} P<0.01,{ }^{* * *} P<0.001$ indicate statistically significant differences

The effect of OA collaterals on hemispheric hemodynamics in patients with severe ICA stenosis is still controversial. Telman et al. reported that collateral circulation through OA had no influence on hemispheric hemodynamics [22]; nevertheless, Henderson et al. found that OA collaterals may be associated with a lower risk of stroke and TIA [23]. In our study, the BFV of ipsilateral MCA was the lowest in the OA group. The possible reason for these inconsistent results may be the different inclusion and grouping criteria. Besides AcoA and PcoA, there may be other collaterals (e.g. leptomeningeal artery) which have little effect on cerebral perfusion. Cheng et al. found cerebral perfusion to be significantly impaired in patients with collateral circulation via secondary collaterals, which was consistent with our findings [24].

\section{Relationship between frontal lobe cognitive function and collateral circulation}

Some studies have proposed that cerebral BFV can reflect the brain perfusion as well as cognitive dysfunctions [25]. Wang et al. reported significant CBF reduction in patients with anterior circulation TIA who had vascular cognitive impairment [26]. Patients with carotid atherosclerosis showed lower scores in verbal fluency test even when the atherosclerosis were subclinical [27], and verbal fluency was improved after carotid artery stenting or carotid endarterectomy for patients with carotid artery stenosis [28]. These findings indicated that CBF was associated with verbal fluency performance. In the present study, decreased performance of Word Fluency Test in the PcoA and OA groups indicated their impaired sematic memory and language ability. The undifferentiated results between the AcoA and control group implied a potential for preseverd language function among AcoA collateral patients. It has been reported that patients with greater carotid artery intima-media thickness had worse TMT performance [29], which is consistent with our findings. Digit Span is a measure of working memory and is not associated with emotional lability [30]. The worse results of TMT and Digit Span in patients with ICA stenosis indicated impaired executive function and working memory included in frontal lobe cognitive function, and the type of collaterals had no significant impact on these subfunctions.

In SCWT, significant difference was only found in RT and AR of SCWT C and SIE, suggesting a higher sensitivity for detection of selective attention in patients with severe ICA stenosis. In SCWT C, subjects needed to suppress the habitual response to the meaning of the words and shift their attention to its incongruent color. The incongruent task in Stroop requires response inhibition, which may be

Table 6 Correlation coefficient between TCD parameters (side of stenosis) and SIE scores

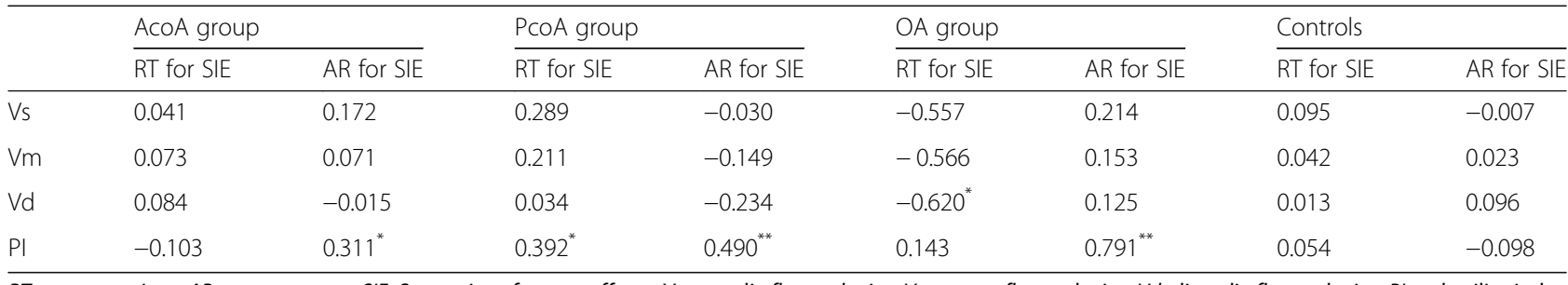

$R T$, response time; $A R$, accuracy rate; $S I E$, Stroop interference effects; $V s$, systolic flow velocity; $V m$, mean flow velocity; $V d$, diastolic flow velocity; $P I$, pulsatility index ${ }^{*} P<0.05,{ }^{* *} P<0.01$ indicate statistically significant correlations 

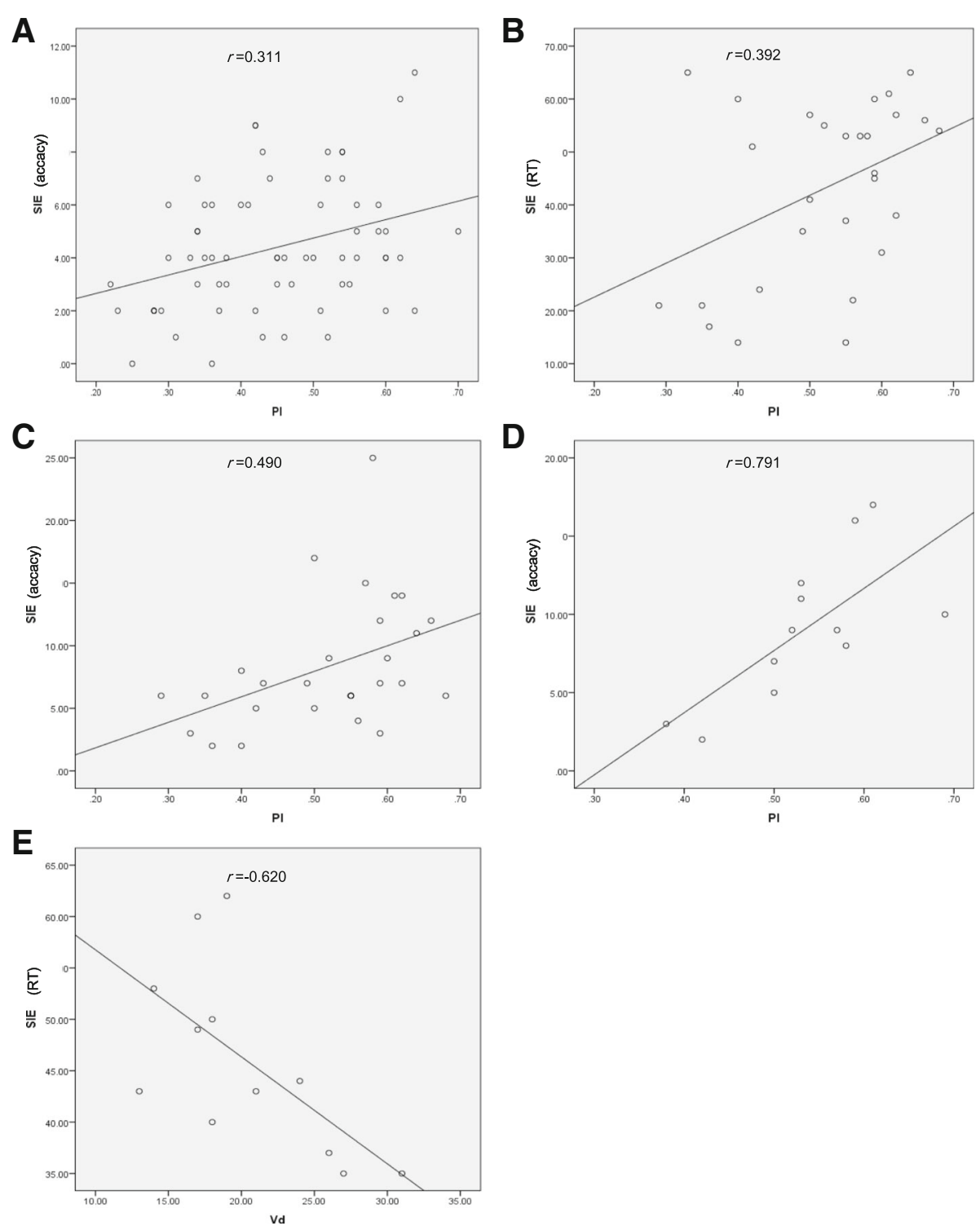

Fig. 1 Correlation between TCD parameters (side of stenosis) and SIE scores. a Scatter plot demonstrating correlation between pulsatility index (PI) and SIE accuracy in the AcoA group. b Scatter plot demonstrating correlation between PI and reaction time of SIE in the PcoA group. $\mathbf{c}$ Scatter plot demonstrating correlation between PI and SIE accuracy in the PcoA group. $\mathbf{d}$ Scatter plot demonstrating correlation between PI and SIE accuracy in the OA group. e Scatter plot demonstrating correlation between diastolic velocity and reaction time of SIE in the OA group

linked with anterior cingulate gyrus [21]. Better performance in SCWT requires more blood flow in frontal lobe [31], and worse performance in patients with ICA stenosis may be explained due to the altered blood flow. Carter et al. hypothesized that the poor performance in SCWT may be associated with the anterior cingulate gyrus dysfunction as evaluated by positron emission tomography [32]. Moreover, Benabarre et al. found the SCWT score to be well correlated with the CBF in striatal, temporomedial, and parietal cortices [33]. Taylor et al. found the activation of left inferior frontal gyrus on positron emission tomography to reflect the Stroop processing [34]. All of the regions mentioned can be affected by reduced blood supply of ICA. Furthermore, the dorsal anterior cingulate cortex receives top-down information from the dorsolateral prefrontal cortex and bottom-up information from the left caudate nucleus [35]. Hypoperfusion may disrupt the interaction of these grey-matter areas, leading to poor performance in SCWT. AcoA has been verified to supply the frontal lobe in patients with ICA occlusion [36]. We also noted patients with collaterals via AcoA exhibited a better performance in SCWT than the patients in other groups. 
Of note, $\mathrm{RT}_{\mathrm{SCWT}} \mathrm{C}$ and $\mathrm{RT}$ for SIE in AcoA group were longer than those of other groups. He et al. found the Chinese language functional areas were located in both frontal and temporal lobes [37]. Therefore, patients in AcoA group may suffer less impairment in language function because of the well-preserved anterior circulation. The normal results of the AcoA group in Word Fluency Test also demonstrated inconsistency. In SCWT, they could be easier to be interfered by the incongruent meaning of the words, and they might spend more time in judging the color of the words. However, there was no difference in AR for SIE only between the AcoA group and controls. We speculate the patients in AcoA group had relatively intact language function manifesting as normal $\mathrm{AR}$ at a cost of prolonged RT. Both RT and AR were increased in PcoA and OA groups, indicating that collaterals via PcoA or OA to be associated with a relatively severe impairment of selective attention. Additionally, $\mathrm{AR}_{\mathrm{SCWT}} \mathrm{C}$ in the $\mathrm{OA}$ group was significantly lower than in normal controls, suggesting the patients in the OA group had more severe selective attention impairment. Reinhard et al. proposed that the cerebral blood autoregulation in patients with collaterals via secondary pathways was worse than that in patients with collaterals via primary pathways [38], and cerebral autoregulation to be crucial for preserving the cognitive function, which may help explain our findings. In general, the frontal lobe cognitive function is impaired to various extents in patients with ICA stenosis. Being different from other frontal lobe cognitive assessment, SCWT, especially SCWT $\mathrm{C}$ and SIE reflected respective position and function for different collaterals more precisely.

\section{Relationship between selective attention and TCD parameters}

We noted a negative correlation between $\mathrm{Vd}$ of MCA and RT for SIE in the OA group, indicating that BFV may be insensitive in reflecting selective attention because we only found statistically significant correlation between Vd and RT for SIE in the OA group. A possible reason is that the inconstant blood flow may be influenced by blood pressure, temperature and psychological state, and cerebral BFV may reflect selective attention only when cerebral perfusion is severely impaired.

The positive correlation between ipsilateral PI and SIE suggests that PI could be a potential indicator for evaluating selective attention impairment. In our patients with severe ICA stenosis, PI of ipsilateral MCA decreased in all patient groups. In previous studies, the relationship between PI and cognitive function remains controversial. Altmann et al. demonstrated that elevated PI was associated with impairment in several cognitive domains [39]. However, Shim et al. found that PI in patients with cognitive impairment did not differ from that in normal controls [40]. We speculate the impaired selective attention may be partly due to the abnormal resistance of cerebral vessels and low cerebral perfusion. Moreover, the more general correlation of PI and AR for SIE indicated AR may be a more valuable indicator in reflecting cognitive function in patients with ICA stenosis.

\section{Limitations}

There are several limitations to the current study. First, the slight statistical difference in diabetes mellitus and hyperlipidemia between the AcoA group and the controls may be due to the limited sample size. However, the worse results of the PcoA and OA group showed the association of diabetes mellitus and hyperlipidemia with the impairment of frontal lobe selection may be nonexistent. Second, the present study was limited by the small sample size of other types of collaterals as only collateral circulation via AcoA, PcoA and OA was analyzed. Third, drugs (especially statins) may be confounding factor on vasomotor reactivity [41]. In the future, we will enlarge the sample size via initiating a multi-center study and take the drug-related confounding factors into account.

\section{Conclusions}

To our knowledge, this study is the first to analyze the influence of different collaterals on CBF and frontal lobe cognitive function in patients with severe ICA stenosis. The frontal lobe cognition function is impaired to different extents according to the type of collaterals. Most notably, selective attention impairment is correlated with the type of collaterals. Collateral circulation via AcoA is associated with a relatively intact selective attention, and collateral circulation via $\mathrm{OA}$ is associated with a severe impairment of selective attention. Furthermore, PI may be a potential indicator for identifying selective attention dysfunction in patients with severe ICA stenosis.

\section{Abbreviations \\ AcoA: Anterior communicating artery; ANOVA: Analysis of variance; AR: Accuracy rate; BFV: Blood flow velocity; CBF: Cerebral blood flow; DSA: Digital subtraction agiography; ICA: Internal carotid stenosis; MCA: Middle cerebral artery; MMSE: Mini-Mental State Examination; NASCET: North American Symptomatic Carotid Endarterectomy Trail; OA: Ophthmaltic artery; PcoA: Posterior communicating artery; PI: Pulsatility index; RT: Response time; SCWT: Stroop Color Word Test; SD: Standard deviation; SIE: Stroop interference effects; TCD: Transcranial Doppler; TIA: Transient ischemic attack; TMT: Trail-Making Test; Vd: Diastolic flow velocity; Vm: Mean flow velocity; Vs: Systolic flow velocity}

\section{Acknowledgements}

Not applicable.

\section{Authors' contributions}

HL and WW designed the study. XD and ZL contributed samples collection. WW and JR collected, analysed and interpreted the data. WW and HL Wrote the initial paper and XY revised the paper. All authors gave intellectual input to the study and approved the final version of the manuscript.

\section{Funding}

This work was supported by research foundation from the Affiliated Hospital of Southwest Medical University (Grant No. 2015-QS-034). The authors 
declare that they have no financial relationship with the organization that sponsored the research, and the funding body was not involved in study design, data collection, analysis and writing of the study.

\section{Availability of data and materials}

The datasets used and/or analysed during the current study are available from the corresponding author on reasonable request.

\section{Ethics approval and consent to participate}

This study was approved by the Ethics Committee of the Affiliated Hospital of Southwest Medical University. All procedures performed in studies involving human participants were in accordance with the ethical standards of the institutional and national research committee and with the 1964 Helsinki declaration and its later amendments or comparable ethical standards. Written informed consent was obtained from all individual participants included in the study.

\section{Consent for publication}

Not applicable.

\section{Competing interests}

The authors declare that they have no competing interests.

\section{Author details}

'Department of Neurology, the Affiliated Hospital of Southwest Medical University, No. 25 Taiping Road, Luzhou 646000, Sichuan, China. ${ }^{2}$ Department of Neurology, People's Hospital of Deyang City, Deyang 618000, Sichuan, China. ${ }^{3}$ Department of Rehabilitation medicine, the Affiliated Hospital of Southwest Medical University, Luzhou 646000, Sichuan, China.

Received: 23 November 2018 Accepted: 26 June 2019

Published online: 05 July 2019

\section{References}

1. Lee JM, Park JM, Min KS, Oh YJ, Kim CJ, Kim YJ. The ameliorative effects of exercise on cognitive impairment and white matter injury from blood-brain barrier disruption induced by chronic cerebral hypoperfusion in adolescent rats. Neurosci Lett. 2017;638:83-9.

2. Zhao JH, Tian XJ, Liu YX, Yuan B, Zhai KH, Wang CW, et al. Executive dysfunction in patients with cerebral hypoperfusion after cerebral angiostenosis/occlusion. Neurol Med Chir (Tokyo). 2013;53:141-7.

3. Sussman ES, Kellner CP, Mergeche $J$, Bruce SS, Mcdowell MM, Heyer EJ, et al. Radiographic absence of the posterior communicating arteries and the prediction of cognitive dysfunction after carotid endarterectomy. J Neurosurg. 2014;121:593-8.

4. Zavoreo I, Bašić KV, Lisak M, Maršić N, Ciliga D, Trošt BT. Cognitive decline and cerebral vasoreactivity in asymptomatic patients with severe internal carotid artery stenosis. Acta Neurol Belg. 2013;113:453.

5. Everts R, Wapp M, Burren Y, Kellner-Weldon F, El-Koussy M, Jann K, et al. Cognitive and emotional effects of carotid stenosis. Swiss Med Wkly. 2014; 144:w13970.

6. Sztriha LK, Nemeth D, Sefcsik T, Vecsei L. Carotid stenosis and the cognitive function. J Neurol Sci. 2009:283:36.

7. Warkentin S, Passant U. Functional imaging of the frontal lobes in organic dementia. Regional cerebral blood flow findings in normals, in patients with frontotemporal dementia and in patients with Alzheimer's disease, performing a word fluency test. Dement Geriatr Cogn Disord. 1997;8:105-9.

8. Reitan RM. Validity of the trail making test as an Indicator of organic brain damage. Percept Motor Skills. 1958;8:271-6.

9. Strasser ES, Haffner P, Fiebig J, Quinlivan E, Adli M, Stamm TJ. Behavioral measures and self-report of impulsivity in bipolar disorder: no association between Stroop test and Barratt impulsiveness Scale. Int J Bipolar Disord. 2016;4:16.

10. Pardo JV, Pardo PJ, Janer KW, Raichle ME. The anterior cingulate cortex mediates processing selection in the Stroop attentional conflict paradigm. Proc Natl Acad Sci U S A. 1990;87:256-9.

11. Saalmann YB, Pinsk MA, Liang W, Xin L, Sabine KJS. The pulvinar regulates information transmission between cortical areas based on attention demands. Science. 2012;337:753-6.
12. Zhang $Y$, Lin $X$, Zhou $H$, Xu J, Du X, Dong GJFiP. Brain Activity toward Gaming-Related Cues in Internet Gaming Disorder during an Addiction Stroop Task. 2016;7.

13. Collaborators NASCET. Beneficial effect of carotid endarterectomy in symptomatic patients with high-grade carotid stenosis. N Engl J Med. 1991; 325:445-53.

14. $X u$ J, Zhu XY, Sun $H, X u X Q, X u S A$, Suo $Y$, et al. Low vitamin $D$ levels are associated with cognitive impairment in patients with Hashimoto thyroiditis. BMC Endocr Disord. 2018;18:87.

15. Spreen O, Strauss E. A compendium of neuropsychological tests: administration, norms, and commentary. Cogn Behav Neurol. 1991;41:1856.

16. Wechsler D. Manual for the WECHSLER adult intelligence SCALE. 1981;70.

17. Wang C, An Y, Yu H, Feng L, Liu Q, Lu Y, et al. Association between exposure to the Chinese famine in different stages of early life and decline in cognitive functioning in adulthood. Front Behav Neurosci. 2016;10:146.

18. Giller CA, Bowman G, Dyer H, Mootz L, Krippner W. Cerebral arterial diameters during changes in blood pressure and carbon dioxide during craniotomy. Neurosurgery. 1993;32:737.

19. Lindegaard KF, Lundar T, Wiberg J, Sjøberg D, Aaslid R, Nornes H. Variations in middle cerebral artery blood flow investigated with noninvasive transcranial blood velocity measurements. Stroke. 1987;18:1025-30.

20. Shin MK. Effects of an exercise program on frontal lobe cognitive function in elders. J Korean Acad Nurs. 2009:39:107-15.

21. Guler S, Utku U, Aynaci O. Early clinical signs, lesion localization, and prognostic factors in unilateral symptomatic internal carotid artery occlusion. J Stroke Cerebrovasc Dis. 2014;23:1908-14.

22. Telman G, Kouperberg E, Sprecher E, Hoffman A, Yarnitsky D. Assessment of ophthalmic artery collateral pathway in the hemispheric cerebral hemodynamics in patients with severe unilateral carotid stenosis. Neurol Res. 2003;25:309-11.

23. Henderson RD, Eliasziw M, Fox AJ, Rothwell PM, Barnett HJ. Angiographically defined collateral circulation and risk of stroke in patients with severe carotid artery stenosis. North American symptomatic carotid endarterectomy trial (NASCET) group. Stroke. 2000;31:128-32.

24. Cheng XQ, Tian JM, Zuo CJ, Liu J, Zhang Q, Lu GM. Quantitative perfusion computed tomography measurements of cerebral hemodynamics: correlation with digital subtraction angiography identified primary and secondary cerebral collaterals in internal carotid artery occlusive disease. Eur J Radiol. 2012:81:1224-30.

25. Becker A, Mandell AR, Tangney JP, Chrosniak LD, Shaw TH. The effects of self-control on cognitive resource allocation during sustained attention: a transcranial Doppler investigation. Exp Brain Res. 2015;233:2215-23.

26. Wang J, Li Y, Bo Z, Jian W, Wang Z, Dan D, et al. Computed tomography perfusion imaging may predict cognitive impairment in patients with firsttime anterior circulation transient ischemic attack. Int J Cardiovasc Imaging. 2016;32:671-7.

27. Wu DM, Zhang JJ, Guo N, Zheng SJ, Zhu QF, Feng YQ, et al. Cognitive impairment correlates with serum carbonyl compound profiles in subclinical carotid atherosclerosis. Neuroreport. 2018;29:1550-7.

28. Kougias P, Collins R, Pastorek N, Sharath S, Barshes NR, Mcculloch K, et al. Comparison of domain-specific cognitive function after carotid endarterectomy and stenting. J Vasc Surg. 2015;62:355-61.

29. Suemoto CK, Santos IS, Bittencourt MS, Pereira AC, Goulart AC, Rundek T, et al. Subclinical carotid artery atherosclerosis and performance on cognitive tests in middle-aged adults: baseline results from the ELSA-Brasil. Atherosclerosis. 2015:243:510-5.

30. Jensen DA, Hovik MF, Monsen NJN, Eggen TH, Eichele H, Adolfsdottir S, et al. Keeping emotions in mind: the influence of working memory capacity on parent-reported symptoms of emotional lability in a sample of children with and without ADHD. Front Psychol. 2018:9:1846.

31. Tsuchiya K, Mitsui S, Fukuyama R, Yamaya N, Fujita T, Shimoda K, et al. An acute bout of housework activities has beneficial effects on executive function. Neuropsychiatr Dis Treat. 2018;14:61-72.

32. Carter CS, Mintun M, Nichols T, Cohen JD. Anterior cingulate gyrus dysfunction and selective attention deficits in schizophrenia: [15O]H2O PET study during single-trial Stroop task performance. Am J Psychiatry. 1997;154:1670.

33. Benabarre A, Vieta E, Martínezarán A, Garciagarcia M, Martín F, Lomeña F, et al. Neuropsychological disturbances and cerebral blood flow in bipolar disorder. Aust N Z J Psychiatry. 2010;40:375-6.

34. Taylor SF, Kornblum S, Lauber EJ, Minoshima S, Koeppe RA. Isolation of specific interference processing in the Stroop task: PET activation studies. Neuroimage. 1997;6:81-92. 
35. Ridderinkhof KR, Wp VDW, Segalowitz SJ, Carter CS. Neurocognitive mechanisms of cognitive control: the role of prefrontal cortex in action selection, response inhibition, performance monitoring, and reward-based learning. Brain Cogn. 2004;56:129-40.

36. de Boorder MJ, Van dGJ, van Dongen AJ, Klijn CJ, Jaap KL, Van Rijk PP, et al. Spect measurements of regional cerebral perfusion and carbondioxide reactivity: correlation with cerebral collaterals in internal carotid artery occlusive disease. J Neurol. 2006;253:1285-91.

37. He C, Andre VG, Gloria G, Pamela T, Andrea H, Duncan JS. Mandarin functionalMRILanguage paradigms. Brain Behav. 2016;6:1-11.

38. Reinhard M, Müller T, Guschlbauer B, Timmer J, Hetzel A. Dynamic cerebra autoregulation and collateral flow patterns in patients with severe carotid stenosis or occlusion. Ultrasound Med Biol. 2003;29:1105-13.

39. Altmann M, Thommessen B, Rønning OM, Benth JŠ, Reichenbach AS, Fure B. Middle cerebral artery Pulsatility index is associated with cognitive impairment in lacunar stroke. J Neuroimaging. 2016;26:431-5.

40. Shim Y, Yoon B, Shim DS, Kim W, An JY, Yang DW. Cognitive correlates of cerebral vasoreactivity on transcranial Doppler in older adults. J Stroke Cerebrovasc Dis. 2015;24:1262-9.

41. Giannopoulos S, Katsanos AH, Tsivgoulis G, Marshall RS. Statins and cerebral hemodynamics. J Cereb Blood Flow Metab. 2012;32:1973-6.

\section{Publisher's Note}

Springer Nature remains neutral with regard to jurisdictional claims in published maps and institutional affiliations.

Ready to submit your research? Choose BMC and benefit from:

- fast, convenient online submission

- thorough peer review by experienced researchers in your field

- rapid publication on acceptance

- support for research data, including large and complex data types

- gold Open Access which fosters wider collaboration and increased citations

- maximum visibility for your research: over $100 \mathrm{M}$ website views per year

At $\mathrm{BMC}$, research is always in progress.

Learn more biomedcentral.com/submissions 